International Journal of Energy and Statistics Vol. 1, No. 4 (2013) 291-292

(C) Institute for International Energy Studies

DOI: $10.1142 / \mathrm{S} 2335680413990019$

\title{
AUTHOR INDEX (Volume 1)
}

Abdollahzade, M., see Iranmanesh, $\mathrm{H}$.

Ahmadi, S., see Fakehi, A. H.

Amiri, S. \& Beneki, C., Bootstrap tests of multinomial populations and its application for resampling energy data

Antonakakis, N. \& Filis, G., Oil prices and stock market correlation: A time-varying approach

Beneki, C. \& Silva, E. S., Analysing and forecasting European union energy data

Beneki, C., see Amiri, S.

Breaker, L. C., Energy production trend extraction using ensemble empirical mode decomposition

Cassiano, K. M., Júnior, L.

A. T., De Souza, R. M., De Menezes, M. L., Pessanha, J. F. M. \& Souza, R. C., Hydroelectric energy forecast

Cassiano, K. M., see Júnior, L. A. T.

Chithambo, L., Firm characteristics and the voluntary disclosure of climate change and greenhouse gas emission information

De Menezes, M. L., see Cassiano, K. M.

De Menezes, M. L., see Júnior, L. A. T.
3 (2013) 155

3 (2013) 171

4 (2013) 225

3 (2013) 215

1 (2013) 17

2 (2013) 127

3 (2013) 215

3 (2013) 195

3 (2013) 205

2 (2013) 113

3 (2013) 205

2 (2013) 113
De Souza, R. M., see

Cassiano, K. M.

3 (2013) 205

Fakehi, A. H., Ahmadi, S. \& Vakili, A., Scenario analysis of Iran's future energy demand and its environmental issues for the year 2041

4 (2013) 225

Filis, G., see Antonakakis, $\mathrm{N}$.

1 (2013) 17

Ghasemi, S. \&

Mahmoudvand, R., A new insight into the failure mode and effects analysis and its application in insurance: A case study in gas refinery

Ghodsi, M., see Qadrdan, M.

Han, A., see Yang, W.

Hassani, H. \&

Mahmoudvand, R., Multivariate singular spectrum analysis: $A$ general view and new vector forecasting approach

1 (2013) 55

Hassani, H., see Iranmanesh, $\mathrm{H}$

Iranmanesh, H.,

Abdollahzade, M., Miranian, A. \& Hassani, H., A developed wavelet-based local linear neuro fuzzy model for the forecasting of crude oil price

3 (2013) 171 
comparison of two approaches for integral purchasing management in an oil-and-gas

company (PETROPARS)

Iranmanesh, S. H., see Shakhsi-Niaei, M.

Júnior, L. A. T., see Cassiano, K. M.

Júnior, L. A. T., De Menezes, M. L., Cassiano, K. M., Pessanha, J. F. M. \& Souza, R. C., Residential electricity consumption forecasting using a geometric combination approach

1 (2013) 1

2 (2013) 143

3 (2013) 205

2 (2013) 113

4 (2013) 281

1 (2013) 1

4 (2013) 257

1 (2013) 55

3 (2013) 171

Papailias, F. \& Thomakos, D. D., Trading energy ETFs with an improved moving average strategy

Papailias, F., see Thomakos, I. D.

Pessanha, J. F. M., see Cassiano, K. M.

Pessanha, J. F. M., see Júnior, L. A. T.

Qadrdan, M., Ghodsi, M. \& Wu, J., Probabilistic wind power forecasting using a single forecast

Sadrabadi, M. R., see Iranmanesh, S. H.

Shakhsi-Niaei, M., Iranmanesh, S. H. \& Torabi, S. A., A review

1 (2013) 31

4 (2013) 243

3 (2013) 205

2 (2013) 113

2 (2013) 99

1 (2013) 1 of mathematical optimization applications in oil-and-gas upstream and midstream management

2 (2013) 143

Shakhsi-Niaei, M., see Iranmanesh, S. H.

1 (2013) 1

2 (2013) 127

Silva, E. S., A combination forecast for energy-related $\mathrm{CO}_{2}$ emissions in the united states

4 (2013) 269

Souza, R. C., see Cassiano, K. M.

3 (2013) 205

Souza, R. C., see Júnior, L. A. T.

2 (2013) 113

Telesca, L. \& Lovallo, M., Fisher-Shannon analysis of wind records

4 (2013) 281

Thomakos, D. D., see

Papailias, F.

Thomakos, I. D. \&

Papailias, F., Momentum trading in New York

Stock Exchange (NYSE) energy stocks

Torabi, S. A., see Shakhsi-Niaei, M.

Vakili, A., see Fakehi, A. H.
Wang, S., see Xie, H.

Wang, S., see Yang, W.

Wu, J., see Qadrdan, M.

Xie, H., Zhang, X. \& Wang, S., The more the better: Forecasting oil price with decomposition-based vector autoregressive model

Yang, W., Han, A. \& Wang, S., Analysis of the interaction between crude oil price and US stock market based on interval data

Zhang, X., see Xie, H.
1 (2013) 31

4 (2013) 243

2 (2013) 143

4 (2013) 225

1 (2013) 45

2 (2013) 85

2 (2013) 99

1 (2013) 45

2 (2013) 85

1 (2013) 45 